\title{
Load Balance vs Energy Efficiency in Traffic Engineering: A Game Theoretical Perspective
}

\author{
Yangming Zhao, Sheng Wang, Shizhong Xu and Xiong Wang \\ School of Communication and Information Engineering \\ University of Electronic Science and Technology of China \\ Chengdu, P. R. China, 611731 \\ E-mail: \{zhaoyangming,wsh_keylab, xsz,wangxiong \}@uestc.edu.cn
}

\author{
Xiujiao Gao and Chunming Qiao \\ Department of Computer Science and Engineering \\ State University of New York at Buffalo \\ Buffalo, NY, USA, 14260-2500 \\ Email: \{xiujiaog, qiao\}@buffalo.edu
}

\begin{abstract}
In this paper, we study the tradeoff between two important traffic engineering objectives: load balance and energy efficiency. Although traditional multi-objective optimization methods can yield a Pareto efficient solution, they need to construct an aggregate objective function (AOF) or model one of the two objectives as a constraint in the optimization problem formulation. As a result, it is difficult to achieve a fair tradeoff between the both objectives. Accordingly, we induce a Nash bargaining framework which treats the two objectives as two virtual players in a game theoretic model, who negotiate how traffic should be routed in order to optimize both objectives. During the negotiation, each of them announces its performance threat value to reduce its cost, so the model is regarded as a threat value game. Our analysis shows that no agreement can be achieved if each player sets its threat value to be its best performance. To avoid such a negotiation break-down, we modify the threat value game to have a repeated process and design a mechanism to not only guarantee an agreement, but also generate a fair solution. In addition, the insights from this work are also useful for achieving a fair tradeoff in other multi-objective optimization problems.
\end{abstract}

Index Terms-Traffic Engineering; Load Balance; Energy Efficiency; Nash Bargaining; Multi-Objective Optimization.

\section{INTRODUCTION}

Many objectives exist in network traffic engineering [1-7], such as minimizing E2E (end to end) delay or hop count, maximizing throughput, balancing link load, and reducing total energy consumption. Most of these objectives are conflicting with each other, in that improving one objective hurts the other. How to achieve a tradeoff between such conflicting objectives is an interesting problem in network traffic engineering. In this paper, we focus on achieving a fair tradeoff between two important traffic engineering objectives: load balance and energy efficiency.

Load balance is a classic objective in traffic engineering [1-4]. The main goal of load balance is to enhance the performance of network traffic while utilizing network resource economically. To achieve load balance, traffic should be distributed among all the links uniformly, so as to reduce the carried traffic on each link, and hence improving the performance of IP traffic, in terms of reduced queueing delay, and enhanced network scalability.

Energy efficiency is a relatively new but increasingly important traffic engineering objective [5-7], whose goal is to save energy consumption even though it might result in unbalanced traffic. More specifically, there are two commonly used models to determine the network energy consumption. One is the sleep energy model, and the other is the speed scaling energy model. In the sleep energy model [8], we should use as a few links to route the traffic as possible, so that the idle links can be put into the sleep mode to save energy consumption. Obviously, this objective contradicts to that of load balance which is to distribute traffic to as many links as possible. Even in the speed scaling energy model [5-7], where the energy consumption of each link is characterized by its energy curve (The energy curve is an non-decreasing function of traffic load on each link. It could be convex or concave depending on its physical architecture. But it is unlike to be the same as congestion cost of each link.), the traffic distribution achieving the highest energy efficiency would hardly be the same as that achieving load balance..

Since carriers/operators are interested in both objectives above instead of only one of them, this question of how to optimally route traffic ean be modeled as a multi-objective optimization problem.

There are two traditional methods to solve the optimization problem with multiple objectives [9]. One is to treat all objectives except the most favorite one as constraints, and then optimize the favorite one. Such a method might work when there were specific performance goals considered desirable for all the other objectives, in the form of the threshold values used to set the corresponding constraints $\boldsymbol{\Lambda}_{\Lambda}$ However, this is often not the case. For example, a carrier does not know (nor wants to set) the desired specific performance of load balance (or energy efficiency). As a result, only the favorite objective will achieve the best performance ot the expense of all the other objectives (due to some ad hoc performance thresholds specified in the corresponding constraints 作 If all the objectives need to be pursued without restricting any to its ad hoc performance threshold, such a method is not suitable.

The other traditional method is to construct an aggregate objective function (AOF), such as the well-known weighted linear sum of the objectives. It will yield a Pareto optimal solution in theory, but it is difficult to determine the appropriate weight for each objective. This is because these objective values have not only different performance metrics representing different dimensions of interest (e.g., load balance and energy efficiency), but also have different scales or orders of magnitude. It seems like that Langarian Relaxation has the same form as AOF, but it should treat one of the objectives as the constraint, which is not suitable to multi-objective optimization in traffie engineering.

Ideally, when we pursue multiple objectives in traffic engineering, we do not want to discriminate against any 
objective by improving the performance of some objectives more significantly than that of the others due to ad hoc constraints or weights assigned for various objectives. In other words, we aim to achieve a fair tradeoff among the objectives we are pursuing under a rational guideline. For instance, from the viewpoint of achieving fairness between multiple objectives, the ones with a relatively larger optimization space should obtain more performance improvement than the ones with a relatively smaller optimization space.

Unfortunately, the traditional multi-objective optimization methods cannot guarantee the fair tradeoffs among different objectives. In the first method mentioned above, all the objectives treated as constraints in the optimization problem are in a weaker position while the one treated as the optimization objective is in a stronger position, resulting in unfair tradeoffs. In the second method mentioned above, the objectives whose values have higher orders of magnitude are likely to be in a stronger position than the objectives that are of lower order of magnitude.

To overcome the difficulty in achieving a fair tradeoff among multiple objectives in traffic engineering, e.g., load balance and energy efficiency, we propose to use a framework based on Nash Bargaining to jointly optimize both objectives and guarantee the fairness between them. We refer to the objectives of load balance and energy efficiency as two virtual game players who are negotiating the solution of traffic engineering. In this framework, we assume that each player changes its threat value (performance threshold) to improve its performance. Accordingly, the interaction of each player can be modeled as a threat value game. Our analysis shows that 1) there are an infinite number of Pareto efficient Nash equilibriums in this threat value game, and 2) a player can improve its performance by unilaterally reducing its threat value. This means that if we were to model this problem as a static game, both players would announce the threat value as low as possible to improve its performance, which would prevent an agreement. To ensure an agreement, we modify the threat value game to be a repeated procedure where each player changes its threat value stepwise. Based on this repeated procedure model, we not only design a

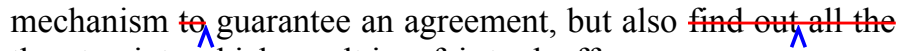
hreat points which result in a fair tradeoff.

The main contributions of our work can be summarized as follows:

- We analyze the problem of achieving a fair tradeoff between multiple incentive incompatible objectives in traffic engineering and provide a useful insight into the general multi-objective optimization problem.

- We also propose a new method to achieve a fair tradeoff between multiple conflicting objectives in traffic engineering. Our method overcomes the difficulty of traditional methods in assigning appropriate priorities (and performance thresholds; to the objectives or determining appropriate weights in the AOF.

The rest of the paper is organized as follows. Section II briefly describes the related work. Section III and Section IV present the commonly used models for load balance and energy efficiency, respectively. In Section V, we introduce the Nash Bargaining theory which forms the foundation of our model and present how to use it in our tradeoff problem. After that, we analyze the tradeoff problem in detail and describe the motivation for using Nash Bargaining to solve the problem in Section VI. In Section VII, we propose a method to achieve a fair tradeoff between load balance and energy efficiency, and pick out all the threat points which can induce a fair solution. We also present case studies of our method in Section VIII and conclude our paper in Section IX.

\section{RELATED WORK}

In the past decades, a lot of works have been done on the traffic engineering in networks. Some have focused on traffic load balance [1-4], while others have tried to minimize the energy consumption [5-7]. Among them, B. Fortzdid a classical work that pursues load balance by optimizing the link weight in the OSPF network [2] and D. Awduche etc. works on achieving load balance with MPLS[10]. Neither of these works, however, considered reducing the energy consumption in the network. On the other hand, recent works in [5] and [11] pursued energy efficiency, but neither of them considers load balance as an objective. To the best of our knowledge, there is no existing work on traffic engineering that pursues both load balance and energy efficiency simultaneously.

To deal with multiple objectives, the commonly used traditional multi-objective optimization approach [9] creates an AOF or treats one of the objectives as the primary objective and expresses all other (secondary) objectives as constraints of the optimization problem. Though both of these methods can yield a Pareto efficient solution, it is difficult to select the appropriate weights when constructing the AOF or to determine the appropriate performance threshold values to be used in the constraints for the secondary objectives. As a result, a fair tradeoff between among the objectives cannot be achieved.

Game theory is a useful tool to solve many network optimization problems. There are an increasing number of researchers who apply it to address routing issues in multilayer network [12-14], cooperation (or competition) among multiple autonomous systems [4], and content provider selection [15]. To the best of our knowledge, there has been no existing work which has analyzed the tradeoff between multiple objectives of a single operator in a game theoretic perspective, let alone any work on achieving fair tradeoff between load balance and energy efficiency.

The work which is most similar to our work is the Nash arbitration scheme [17]. In this scheme, an AOF having the same form as that in Nash bargaining was introduced to derive the optimization solution. But it approached the problem mostly from a multi-objective optimization perspective while we will approach it from a game theoretic perspective in this paper. In addition, [16] only proved that the solution of their method depends on the threat value of each objective and an objective that is the farthest away from its threat value tends to improve most significantly. However, it did not show how the threat value will affect the outcome of the optimization, nor how to set the threat values.

\section{LOAD BALANCE MODEL}

In this section, we describe the network model and formulate the standard load balance optimization problem. 
Consider a network represented by a directed graph $\boldsymbol{G}=(\boldsymbol{V}, \boldsymbol{E})$, where $\boldsymbol{V}$ denotes the set of nodes and $\boldsymbol{E}$ denotes the set of directed physical links. Let $\boldsymbol{P}_{i j}=\left\{p_{i j}^{k}\right\}$ denote the set of all the paths from $i$ to $j$, where $p_{i j}^{k}$ denote the $k^{\text {th }}$ path from $i$ to $j$ and $i, j$ $\in \boldsymbol{V}$. A link $l$ on the $k^{\text {th }}$ path from $i$ to $j$ will be referred to as $l \in p_{i j}^{k}$. We also use $x_{i j}^{k}$ to denote the rate of flow on the $k^{\text {th }}$ path from $i$ to $j, x_{l}$ the rate of flow on link $l \in \boldsymbol{E}$, and $d_{i j}$ the demand from $i$ to $j$. The capacity of link $l \in \boldsymbol{E}$ is $c_{l}>0$.

The goal of load balance is to enhance the network performance, such as low congestion cost and high scalability. In practice, network operators control routing either by changing OSPF link weights [2] or by establishing MPLS label-switched paths [10]. The latter one is assumed in our model. It is not only because it is optimal, i.e. it gives the routing with minimum congestion cost, but also due to the fact that it can be realized easily by routing protocols that use MPLS tunneling.

Based on the above discussion, the traffic engineering for load balance can be formulated as follows:

$$
\begin{array}{ll}
\text { minimize } & \sum_{l} f_{l}\left(x_{l}\right) \\
\text { subject to } & x_{l}=\sum_{i, j: i \neq j} \sum_{k: l \in p_{i j}^{k}} x_{i j}^{k}, \quad \forall l \in E \\
& x_{l} \leq c_{l} \quad \forall l \in E \\
& \sum_{k} x_{i j}^{k}=d_{i j} \quad \forall i, j \neq i
\end{array}
$$

where $f_{l}(\cdot)$ represents the congestion cost of link $l \in \boldsymbol{E}$. In this paper, we assume that $f_{l}(\cdot)$ is a convex, continuous and non-decreasing function of $x_{l}$. Using such a cost function in the optimization objective will penalize high link utilization and balance the load in the networks. A queuing theory style congestion cost function such as $f_{l}\left(x_{l}\right)=x_{l} /\left(c_{l}-x_{l}\right)$ is usually adopted for this purpose, as it has the desired properties of being convex, continuous and non-decreasing with $x_{l}$. By using this congestion cost function, the link has higher congestion cest at higher utilization level than the eost of links at the lower utilization level $\lambda$ so that traffic should ${ }_{\lambda}$ be distributed uniformly in the network to achiev the optimal solution. Therefore, queuing theory style congestion-cost funetion result in load balanee solution.

\section{ENERGY EFFICIENCY MODEL}

While the load balance problem usually assumes that the congestion cost function of each link is a convex, continuous and non-decreasing function of the amount of traffic carried by the link, there are two popular models that relate power consumption to traffic load: speed scaling and powering down. In the former model, the processing (or transmission) speed of a network element is adjusted (and accordingly, the corresponding energy consumption also varies) according to the carried traffic load. In the latter model, one tries to turn down any elements carrying no traffic load at all to save energy

We focus on the speed scaling model in this paper because it is more realistic. In addition, the powering down model focuses on optimizing an individual element in isolation [5], but we want to examine optimization problems that arise in a network consisting of multiple network elements. In particular, we assume that the energy consumption in the network can all be represented in terms of the energy consumption of the links, which can be characterized by energy curve $g_{l}\left(x_{l}\right)$. The goal of traffic engineering to achieve energy efficiency is thus to minimize the total energy cost of all the links in the network. Accordingly, the optimization problem can be formulated as follows:

minimize

$$
\begin{aligned}
& \sum_{l} g_{l}\left(x_{l}\right) \\
& x_{l}=\sum_{i, j: i \neq j} \sum_{k: l \in p_{i j}^{k}} x_{i j}^{k}, \quad \forall l \in E \\
& x_{l} \leq c_{l} \quad \forall l \in E \\
& \sum_{k} x_{i j}^{k}=d_{i j} \quad \forall i, j \neq i
\end{aligned}
$$

Note that, the energy curve is often modeled by a polynomial function $g_{l}\left(x_{l}\right)=\mu_{l} x_{l}^{\alpha}$, where $\mu_{l}$ and $\alpha$ are device specific parameters. For example, the value of the $\alpha$ is $1.11,1.66$, and 1.62 for Intel PXA 270, a TCP offload engine, and Pentium M 770 , respectively [8].

\section{NASH BARGAINING}

Since our method to trade off between load balance and energy efficiency is based on the Nash Bargaining solution [17], we briefly introduce the Nash Bargaining solution and analyze its properties in this section.

Consider two players, labeled $i=1,2$, that are trying to achieve an agreement over a strategy space $X$. And the utility function $u_{i}$ of each player $i$ is defined over the space $X \cup\{T\}$, where $T$ is the strategy of the two players that leads to a failed agreement. Define the space $S$ to be the set of all possible utilities that the two players can achieve, i.e. ,

$$
S=\left\{\left(u_{1}(x), u_{2}(x)\right) \mid x=\left(x_{1}, x_{2}\right) \in X\right\}
$$

Let $d=\left(u_{1}\left(t_{1}, t_{2}\right), u_{2}\left(t_{1}, t_{2}\right)\right)=\left(d_{1}, d_{2}\right)$ be the pair of utility expected to be obtained by the two players when they fail to achieve an agreement, i.e. the disagreement point or threat point. We also say $d_{1}$ and $d_{2}$ are the threat values of play 1 and player 2 , respectively.

A bargaining problem is defined as the pair $(S, d)$ where $S \subset R^{2}$ and $d \in S$ such that

\section{- $S$ is a convex and compact set}

- There is some $s \in S$ such that $s>d$, by which we mean $s_{i} \geq d_{i}$ for $i=1,2$ and $s_{i}>d_{i}$ for $i=1$ or 2 .

The Nash Bargaining solution we are interested in is a mapping $f:(S, d) \rightarrow S$ for every bargaining problem $(S, d)$ (note that $f_{i}(S, d)$ is used to represent the utility value of player $i$ ) which satisfies the following four properties:

1. Pareto efficiency: A bargaining solution $f(S, d)$ is Pareto-efficient means that there is no point $\left(s_{1}, s_{2}\right) \in S$ such that $s_{i} \geq f_{i}(S, d)$ for all $i$ and $s_{i}>f_{i}(S, d)$ for some $i$.

2. Symmetry: If $(S, d)$ is symmetric around $s_{1}=s_{2}$, i.e. $\left(s_{1}, s_{2}\right)$ $\in S$ iff $\left(s_{2}, s_{1}\right) \in S$ and $d_{1}=d_{2}$, then $f_{1}(S, d)=f_{2}(S, d)$.

3. Invariance to equivalent utility representation: Assume the solution of Nash Bargaining $(S, d)$ is $\left(s_{1}, s_{2}\right)$, if it is transformed to $\left(S^{\prime}, d^{\prime}\right)$ by taking $s_{i}{ }^{\prime}=\alpha_{i} s_{i}+\beta_{i}$ and $d_{i}{ }^{\prime}=\alpha_{i} d_{i}+\beta_{i}$, where $\alpha_{i}>0$, the solution of $\left(S^{\prime}, d^{\prime}\right)$ is $\left(\alpha_{1} s_{1}+\beta_{1}, \alpha_{2} S_{2}+\beta_{2}\right)$.

4. Independence of irrelevant alternatives: Given two bargaining problem $(S, d)$ and $\left(S^{\prime}, d\right)$, where $S^{\prime} \subset S$, if $f(S, d)$ 
$\in S^{\prime}$, there must be $f(S, d)=f\left(S^{\prime}, d\right)$.

Nash's result [16] shows that there is a unique bargaining solution that satisfies the four properties, which is the solution of the following optimization problem:

$$
\begin{array}{ll}
\operatorname{maximize} & \left(s_{1}-d_{1}\right)\left(s_{2}-d_{2}\right) \\
\text { subject to } & \left(s_{1}, s_{2}\right) \in S \\
& \left(s_{1}, s_{2}\right) \geq\left(d_{1}, d_{2}\right)
\end{array}
$$

If the utility function of each player is defined to be the opposite number of its cost function, the Nash Bargaining form of our problem can be presented as:

$$
\begin{array}{ll}
\text { maximize } & \left(d_{L B}-\sum_{l \in E} f_{l}\left(x_{l}\right)\right)\left(d_{E E}-\sum_{l \in E} g_{l}\left(x_{l}\right)\right) \\
\text { subject to } & x_{l}=\sum_{i, j: i \neq j} \sum_{k: l \in p_{i j}^{k}} x_{i j}^{k}, \quad \forall l \in E \\
& x_{l} \leq c_{l} \quad \forall l \in E \\
& \sum_{k} x_{i j}^{k}=d_{i j} \quad \forall i, j \neq i \\
& \sum_{l \in E} f_{l}\left(x_{l}\right) \leq d_{L B} \\
& \sum_{l \in E} g_{l}\left(x_{l}\right) \leq d_{E E}
\end{array}
$$

where $d_{L B}$ and $d_{E E}$ are the performance thresholds (maximal cost tolerated by each player) set by the load balance and energy efficiency objectives, respectively. It should be noted that these performance thresholds are-different from that we mentioned previously. The thresholds can be the performance in the worst case while it is impossible in the commonly used multi-objective optimization problems. What is more, we will discuss how to set these thresholds to derive a fair solution.

\section{Problem Analysis}

In this section, we will analyze the desirable properties of the solution obtained by our method and explain why the Nash Bargaining framework is suitable to our problem. For each of the optimization problems in (1) and (2), $x_{l}$ in the objective function can be substituted based on the first equation (constraint), so $f_{l}(\cdot)$ (and $g_{l}(\cdot)$ ) can be treated as a function of $x=\left\{x_{i j}^{k}\right\}$. From now on, we also use $f_{l}(x)$ and $g_{l}(x)$ to denote the cost function of load balance and energy efficiency for link $l$ hereafter.

\section{A. Desirable Properties}

a. Pareto efficiency: In our problem, the two objectives (load balance and energy efficiency) are pursued by one operator, so that the solution $x *$ should not be worse than any solution $x$ for both objectives, i.e. there exist no feasible solution $x$ such that $\sum_{l \in E} f_{l}(x)<\sum_{l \in E} f_{l}\left(x^{*}\right)$ and $\sum_{l \in E} g_{l}(x)<\sum_{l \in E} g_{l}\left(x^{*}\right)$. It also means that the solution of our method should lie on the Pareto frontier (See Definition 6) and hence no other solution can improve at least one player's performance without hurting the performance of the other one.

b. Fairness: Load balance and energy efficiency are both pursued and any one of them are not preferred more than the other one, so that we should treat them equitably. The fairness is defined as follows:

Definition 1: Let $x_{L B}$ and $x_{E E}$ be the solutions to the optimization problems (1) and (2) respectively, then we define the best case and worst case load balance cost to be

$$
L B_{\text {best }}=\sum_{l \in E} f_{l}\left(x_{L B}\right) \text { and } L B_{\text {worst }}=\sum_{l \in E} f_{l}\left(x_{E E}\right)
$$

respectively. Similarly, we define the best case and worst case costs of energy efficiency to be

$$
E E_{\text {best }}=\sum_{l \in E} g_{l}\left(x_{E E}\right) \text { and } E E_{\text {worst }}=\sum_{l \in E} g_{l}\left(x_{L B}\right)
$$

respectively.

Definition 2 (Proportional Fairness): Assume that $s_{L B}$ and $s_{E E}$ are the objective values of load balance and energy efficiency corresponding to a solution that achieves some tradeoffs between the two, then the solution is proportionally fair if and only if it satisfies the following equation:

$$
\frac{L B_{\text {worst }}-s_{L B}}{L B_{\text {worst }}-L B_{\text {best }}}=\frac{E E_{\text {worst }}-s_{E E}}{E E_{\text {worst }}-E E_{\text {best }}} \text {. }
$$

Definition 2 means that in the solution with a fair tradeoff, both the load balance and energy efficiency objectives obtain the same percentage (or relative) improvement. It is worth nothing that although the values of the two utility functions may have significantly different orders of magnitude, and/or their optimization spaces have different sizes, the above definition of a fair tradeoff uses a relative term and as a result, each objective function will result in a proportional improvement over its worst case performance.

Definition 3 (Max-Min Fairness): Assume that $s_{L B}$ and $s_{E E}$ are the objective values of load balance and energy efficiency corresponding to a solution that achieves some tradeoffs between the two, then, the solution is max-min fair iff

$$
\left(s_{L B}, s_{E E}\right)=\arg \max _{\left(s_{1}, s_{2}\right) \in S} \min \left\{\frac{L B_{\text {worst }}-s_{1}}{L B_{\text {worst }}-L B_{\text {best }}}, \frac{E E_{\text {worst }}-s_{2}}{E E_{\text {worst }}-E E_{\text {best }}}\right\} .
$$

In other words, a Max-Min fair solution maximizes the relative performance improvement of the objective who gets less relative performance improvement, and accordingly, tries to minimize the performance gap between the two objective functions (in terms of their relative performance improvement). Theorem 1: For a Pareto efficient solution $\left(s_{L B}, s_{E E}\right)$, if this solution is proportional fair, it must also be max-min fair.

\section{Proof:}

We prove it by contradiction by assuming that a Pareto efficient solution $\left(s_{L B}, s_{E E}\right)$ is proportional fair but not max-min fair.

Let another Pareto solution $\left(s_{L B}^{\prime}, s_{E E}^{\prime}\right) \neq\left(s_{L B}, s_{E E}\right)$ be max-min fair solution such that

$$
\begin{aligned}
& \min \left\{\frac{L B_{\text {worst }}-s_{L B}^{\prime}}{L B_{\text {worst }}-L B_{\text {best }}}, \frac{E E_{\text {worst }}-s_{E E}^{\prime}}{E E_{\text {worst }}-E E_{\text {best }}}\right\}> \\
& \min \left\{\frac{L B_{\text {worst }}-s_{L B}}{L B_{\text {worst }}-L B_{\text {best }}}, \frac{E E_{\text {worst }}-s_{E E}}{E E_{\text {worst }}-E E_{\text {best }}}\right\}=, \\
& \frac{L B_{\text {worst }}-s_{L B}}{L B_{\text {worst }}-L B_{\text {best }}}=\frac{E E_{\text {worst }}-s_{E E}}{E E_{\text {worst }}-E E_{\text {best }}}
\end{aligned}
$$

the following inequations must be satisfied

$$
\begin{gathered}
\frac{L B_{\text {worst }}-s_{L B}^{\prime}}{L B_{\text {worst }}-L B_{\text {best }}}>\frac{L B_{\text {worst }}-s_{L B}}{L B_{\text {worst }}-L B_{\text {best }}} \\
\text { and } \frac{E E_{\text {worst }}-s_{E E}^{\prime}}{E E_{\text {worst }}-E E_{\text {best }}}>\frac{E E_{\text {worst }}-s_{E E}}{E E_{\text {worst }}-E E_{\text {best }}} .
\end{gathered}
$$


These two inquations imply that $\left(s_{L B}, s_{E E}\right)$ is not a Pareto efficiency solution, which is a contradiction.

Theorem 1 means that we can focus on finding a tradeoff solution which is Pareto efficient and satisfies the proportional fairness property hereafter.

\section{B. Why Nash Bargaining}

The key idea of our work is to find a utility allocation method such that the fairness between multiple objectives can be guaranteed. We adopt the Nash Bargaining framework because it not only is a classical cooperative game framework which pursues the fairness between the players in the game, but also will obtain a Pareto efficient solution. Since a solution using Nash Bargaining is determined by the threat points which can be treated as the performance thresholds of each player, a key issue is to determine the threat point of a Nash Bargaining problem such that the fairness between different objectives can be guaranteed.

\section{TRADING OFF LOAD BALANCE AGAINST ENERGY EFFICIENCY USING NASH BARGAINING}

In this section, we realize the tradeoff between load balance and energy efficiency based on Nash Bargaining framework. In such a framework, each player announces its threat value to improve its own performance, so that we call it threat value game. In subsection VII.A, we introduce this game and analyze it in depth. Our analysis shows that such game has an infinite number of Nash equilibriums and each player selfishly determines threat value will prevent the agreement. To avoid this problem, we modify the threat value game to be a repeated process and design a mechanism to guarantee the agreement in subsection VII.B. Through this mechanism we can easily getall the threat points which can result in fair solution. Bue tg the optimization problem (5) is not in a convex form, we show how to translate it into a convex form which can be solved more efficiently in subsection VIII.C.

\section{A. Nash Bargaining Model and Threat Value Game}

Let $a_{L B}$ and $a_{E E}$ be the performance threshold of load balance and energy efficiency, respectively. The Nash Bargaining solution can be derived by the following optimization problem: maximize $\quad\left(a_{L B}-\sum_{l \in E} f_{l}\left(x_{l}\right)\right)\left(a_{E E}-\sum_{l \in E} g_{l}\left(x_{l}\right)\right)$ subject to

$$
\begin{aligned}
& x_{l}=\sum_{i, j: i \neq j} \sum_{k: l \in p_{i j}^{k}} x_{i j}^{k}, \quad \forall l \in E \\
& x_{l} \leq C_{l} \quad \forall l \in E \\
& \sum_{k} x_{i j}^{k}=d_{i j} \quad \forall i, j \neq i \\
& \sum_{l \in E} f_{l}\left(x_{l}\right) \leq a_{L B} \\
& \sum_{l \in E} g_{l}\left(x_{l}\right) \leq a_{E E}
\end{aligned}
$$

Obviously, both players can change its threat value to improve its performance. But neither of them can change the threat value arbitrarily, because it may prevent the agreement (no feasible solution to (5)). To analyze such a game in more depth, we first have the following definition.

Definition 4: A threat value game is a tuple $\boldsymbol{G}=\left(N,\left(A_{i}\right)_{i \in\{L B, E E\}}\right.$, $\left.\left(c_{i}\right)_{i \in\{L B, E E\}}\right)$, where
$A_{i}$ is the set of available strategies for player $i \in\{L B, E E\}$. In our model, $A_{L B}=\left[L B_{\text {best }}, L B_{\text {worst }}\right]$ and $A_{E E}=\left[E E_{\text {best }}, E E_{\text {worst }}\right]$. We use $a_{i}$ to denote a special strategy for player $i$.

$c_{i}$ is the cost for player $i \in\{L B, E E\}$. The value of $c_{i}$ depends on the solution of optimization problem (5).

The threat value can also be treated as the performance threshold of each player to sign the agreement. If there exist no feasible solution to (5) (which means no agreement can be achieved), $c_{i}=\infty$ for each player. Otherwise,

$$
c_{L B}^{*}=\sum_{l \in E} f_{l}\left(x^{*}\right) \text { and } c_{E E}^{*}=\sum_{l \in E} g_{l}\left(x^{*}\right)
$$

where $x^{*}=\left\{x^{k}{ }_{i j}^{*}\right\}$ is the solution of (5).

Lemma 1: For each player in the threat value game, reducing its threat value unilaterally will improve its performance or prevent the agreement.

Proof:

Without loss of generality, we assume that player energy efficiency reduces its threat value unilaterally, and as a result, the threat point is moved from $\left(a_{L B}, a_{E E}\right)$ to $\left(a_{L B}, a_{E E}\right)$, where $a_{E E}>a_{E E}$.

If there exists no feasible solution for the threat point $\left(a_{L B}\right.$, $a_{E E}^{\prime}$ ), the agreement will be broken, and the cost for both players is $\infty$. Otherwise, we denote the optimal solution before and after player energy efficiency reduces its threat value by $x$ and $x$ ' respectively, then we know:

$$
\begin{aligned}
& \left(a_{L B}-\sum_{l \in E} f_{l}(x)\right)\left(a_{E E}-\sum_{l \in E} g_{l}(x)\right)> \\
& \left(a_{L B}-\sum_{l \in E} f_{l}\left(x^{\prime}\right)\right)\left(a_{E E}-\sum_{l \in E} g_{l}\left(x^{\prime}\right)\right) \\
& \left(a_{L B}-\sum_{l \in E} f_{l}(x)\right)\left(a_{E E}^{\prime}-\sum_{l \in E} g_{l}(x)\right)< \\
& \left(a_{L B}-\sum_{l \in E} f_{l}\left(x^{\prime}\right)\right)\left(a_{E E}^{\prime}-\sum_{l \in E} g_{l}\left(x^{\prime}\right)\right)
\end{aligned}
$$

and

from $(6.1) /(6.2)$

$$
\frac{a_{E E}-\sum_{l \in E} g_{l}(x)}{a_{E E}^{\prime}-\sum_{l \in E} g_{l}(x)}>\frac{a_{E E}-\sum_{l \in E} g_{l}\left(x^{\prime}\right)}{a_{E E}^{\prime}-\sum_{l \in E} g_{l}\left(x^{\prime}\right)}
$$

(6.3) can be converted to be

$$
\left(a_{E E}^{\prime}-a_{E E}\right)\left(\sum_{l \in E} g_{l}\left(x^{\prime}\right)-\sum_{l \in E} g_{l}(x)\right)>0
$$

Since $a_{E E}>a_{E E}$, we obtain

$$
\sum_{l \in E} g_{l}(x)>\sum_{l \in E} g_{l}\left(x^{\prime}\right)
$$

Definition 5: Let $S$ be the set of all the possible cost pairs for two players. We say that the cost pair $\left(a_{L B}, a_{E E}\right) \in S$ is dominated by $\left(a_{L B}, a_{E E}^{\prime}\right) \in S$ iff:

$a_{L B} \geqslant a_{L B}^{\prime}$ and $a_{E E} \geqslant a_{E E}^{\prime}$
$a_{L B}>a_{L B}^{\prime}$ or $a_{E E}>a_{E E}^{\prime}$

Definition 6: A Pareto frontier is a subset of $S$, such that all the points in the Pareto frontier are not dominated by any points in $Y$.

Theorem 2: Every point $\left(a_{L B}, a_{E E}\right)$ in Pareto frontier is Nash equilibrium in the threat value game.

\section{Proof:}

From lemma 1, we know that if a player increase its threat value unilaterally will increase its cost, so that it is cost inefficient for any player to increase its threat value unilaterally. 
On the other hand, if a player reduces its threat value, there will be no feasible solution, which can be proven by contradiction as follows:

Without loss of generality, we assume that player energy efficiency reduce its threat value to $a_{E E}^{\prime}<a_{E E}$ and there exists a feasible solution $x$. From lemma 1, we know that

$$
\sum_{l \in E} g_{l}\left(x^{\prime}\right)<\sum_{l \in E} g_{l}(x) .
$$

Combining with (6.1), we have

$$
\sum_{l \in E} f_{l}\left(x^{\prime}\right)>\sum_{l \in E} f_{l}(x)
$$

where $x$ is the optimal solution before the player energy efficiency reduces its threat value. Since $\left(a_{L B}, a_{E E}\right)$ is not dominated by any point in $S$,

and

$$
\begin{aligned}
& a_{L B}=\sum_{l \in E} f_{l}(x) \\
& a_{E E}=\sum_{l \in E} g_{l}(x)
\end{aligned}
$$

must be satisfied; Otherwise $\left(a_{L B}, a_{E E}\right)$ will be dominated by the cost pair associated with the solution of (5).

Substituting (7.2) into (7.1) leads to

$$
\sum_{l \in E} f_{l}\left(x^{\prime}\right)>a_{L B}
$$

which contradicts to the constraint of (5).

\section{B. How to derive a fair solution}

From temma 1, when each player determines its performance selfishly, each of them wants to set its threat value to be the minimal value in its strategy space. Unfortunately, the threat value combined with the best performance of each player will make the optimization problem in (5) infeasible and prevent the agreement achievement. In order to avoid such an undesirable situation, we modify the model formulated in the previous subsection to be a repeated Nash Bargaining problem as shown in Fig.1.

In this repeated Nash Bargaining model, each player changes its threat value stepwise to optimize it performance. Let $\left(a^{(k)}{ }_{L B}\right.$, $a_{E E}^{(k)}$ ) denote the threat point during the $k^{\text {th }}$-iteration, and $x^{(k)}$ be the optimal solution of Nash Bargaining,. We assume that each player updates its threat value during the $k^{\text {th }}$-iteration as a function of its current threat value $\left(a^{(k)}{ }_{L B}\right.$ for player LB and $a^{(k)}{ }_{E E}$ for player EE) and the optimal solution of Nash Bargaining $\left(x^{(k)}\right)$. Therefore, we have,

$$
\begin{aligned}
& a_{L B}^{(k+1)}=h_{L B}\left(a_{L B}^{(k)}, x^{(k)}\right) \\
& a_{E E}^{(k+1)}=h_{E E}\left(a_{E E}^{(k)}, x^{(k)}\right)
\end{aligned}
$$

To guarantee the fairness of the solution, we first find a threat point to induce a fair solution as follows.

Theorem 2: If the cost function of both load balance and energy efficiency, i.e. $f_{l}(\cdot)$ and $g_{l}(\cdot)$ for all $l \in E$, are continuous, then the threat point of Nash Bargaining (5) ( $\left.L B_{\text {worst }}, E E_{\text {worst }}\right)$ will yield a fair solution.

\section{Proof:}

Let $x^{*}=\left\{x_{l}^{*}\right\}$ be the solution of (5) with $\left(a_{L B}, a_{E E}\right)=\left(L B_{\text {worst }}\right.$, $\left.E E_{\text {worst }}\right)$ and we denote the cost of load balance and energy efficiency in the solution of such a bargaining problem by $s_{L B}$ and $s_{E E}$ respectively.

Construct a new bargaining problem $\left(S^{\prime}, d^{\prime}\right)$, by setting $s_{E E}^{\prime}=\alpha s_{E E}+\beta$ and $d_{E E}^{\prime}=\alpha d_{E E}+\beta$, where

$$
\alpha=\frac{L B_{\text {worst }}-L B_{\text {best }}}{E E_{\text {worst }}-E E_{\text {best }}} \text { and } \beta=\frac{L B_{\text {best }} E E_{\text {worst }}-L B_{\text {worst }} E E_{\text {best }}}{E E_{\text {worst }}-E E_{\text {best }}}
$$

then the cost of load balance and energy efficiency in $\left(S^{\prime}, d^{\prime}\right)$ are denoted by $s_{L B}^{\prime}$ and $s_{E E}^{\prime}$ respectively.

In such bargaining problem, we have

$$
E E_{\text {wosst }}^{\prime}=L B_{\text {worst }}^{\prime} \text { and } E E_{\text {best }}^{\prime}=L B_{\text {best }}^{\prime} \text {. }
$$

where $L B{ }^{\prime}{ }_{\text {best }}$ and $L B^{\prime}{ }_{\text {worst }}$ (or $E E^{\prime}{ }_{\text {best }}$ and $E E^{\prime}{ }_{\text {worst }}$ ) are in the best and worst case load balance (or energy efficiency) cost problem respectively. Due to the fact that $f_{l}(\cdot)$ and $g_{l}(\cdot)$ are continuous for all $l \in E$, the cost function of load balance and energy efficiency have the same range in $\left(S^{\prime}, d^{\prime}\right)$.

If $\left(y_{L B}, y_{E E}\right)$ is a feasible solution of $\left(S^{\prime}, d^{\prime}\right)$, then we consider the following equation group

$$
\left\{\begin{array}{l}
\sum_{l \in E} f_{l}\left(x_{l}\right)=y_{E E} \\
\sum_{l \in E} g_{l}\left(x_{l}\right)=y_{L B} \\
\sum_{k} x_{i j}^{k}=d_{i j} \quad \forall i, j \neq i \\
x_{l}=\sum_{i, j: i \neq j} \sum_{k: l \in p_{i j}^{k}} x_{i j}^{k}, \quad \forall l \in E
\end{array}\right.
$$

Because there are $|E|+\sum_{i=1}^{|V|} \sum_{j=1, j \neq i}^{|V|}\left|\left\{p_{i j}^{k}\right\}\right|$ variables but only

$2+|\boldsymbol{E}|+|\boldsymbol{V}|(|\boldsymbol{V}|-1)$ constraints in equation group (9), a solution must exist. It means that $\left(y_{E E}, y_{L B}\right)$ is also a feasible outcome of $\left(S^{\prime}, d^{\prime}\right)$. Hence, $s^{\prime}{ }_{L B}=S^{\prime}{ }_{E E}$ must be satisfied in $\left(S^{\prime}, d^{\prime}\right)$. Based on the Nash Bargaining's property of invariance to equivalent utility representation, we obtain

$$
\begin{aligned}
& L B_{\text {best }}=\alpha E E_{\text {best }}+\beta \\
& s_{L B}=\alpha s_{E E}+\beta \\
& L B_{\text {worst }}=\alpha E E_{\text {worst }}+\beta
\end{aligned}
$$

From (10.3)-(10.2),

$$
L B_{\text {worst }}-s_{L B}=\alpha\left(E E_{\text {worst }}-s_{E E}\right)
$$

Similarly, (10.3)-(10.1) can yield

$$
L B_{\text {worst }}-L B_{\text {best }}=\alpha\left(E E_{\text {worst }}-E E_{\text {best }}\right)
$$

From $(11.1) /(11.2)$, we obtain

$$
\frac{L B_{\text {worst }}-s_{L B}}{L B_{\text {worst }}-L B_{\text {best }}}=\frac{E E_{\text {worst }}-s_{E E}}{E E_{\text {worst }}-E E_{\text {best }}}
$$

Theorem 2 gives an initial threat point which can guarantee the fairness of a Nash Bargaining solution. The following mechanism is designed to prevent players deviating from such a fair solution when they are optimizing its performance selfishly by changing its threat value.

Mechanism 1: Let $\left(a_{L B}^{(k)}, a_{E E}^{(k)}\right)$ denote the threat point during $k^{\text {th }}$-iteration and $x^{(k)}$ be the optimal solution of Nash Bargaining, we initialize the threat point at ( $\left.L B_{\text {worst }}, E E_{\text {worst }}\right)$ and constraint the threat value of each player to satisfy

$$
\begin{aligned}
& a_{L B}^{(k+1)} \geq a_{L B}^{(k)}-\frac{a_{L B}^{(k)}-\sum_{l \in E} f_{l}\left(x^{(k)}\right)}{2}=\frac{1}{2}\left(a_{L B}^{(k)}+\sum_{l \in E} f_{l}\left(x^{(k)}\right)\right) \\
& a_{E E}^{(k+1)} \geq a_{E E}^{(k)}-\frac{a_{E E}^{(k)}-\sum_{l \in E} g_{l}\left(x^{(k)}\right)}{2}=\frac{1}{2}\left(a_{E E}^{(k)}+\sum_{l \in E} g_{l}\left(x^{(k)}\right)\right)
\end{aligned}
$$


With the above two constraints, each player can only claim to occupy half of the performance gap between its threat value and the Nash Bargaining solution.

Lemma 2: Let $x^{*}$ be the optimal solution of optimization problem (5) associated with threat point $\left(a_{L B}, a_{E E}\right)$, then $x^{*}$ is also the optimal solution of problem (5) associated with the threat $\operatorname{point}\left(\frac{1}{2}\left(a_{L B}+\sum_{l \in E} f_{l}\left(x^{*}\right)\right), \frac{1}{2}\left(a_{E E}+\sum_{l \in E} g_{l}\left(x^{*}\right)\right)\right)$.

\section{Proof:}

$x^{*}$ is a feasible solution for the Nash Bargaining problem when the threat point is $\left(a_{L B}, a_{E E}\right)$, so

$$
\sum_{l \in E} f_{l}\left(x^{*}\right) \leq a_{L B}
$$

then

$$
\sum_{l \in E} f_{l}\left(x^{*}\right) \leq \frac{1}{2}\left(a_{L B}+\sum_{l \in E} f_{l}\left(x^{*}\right)\right)
$$

Similarly,

$$
\sum_{l \in E} g_{l}\left(x^{*}\right) \leq \frac{1}{2}\left(a_{E E}+\sum_{l \in E} g_{l}\left(x^{*}\right)\right)
$$

The above means that $x^{*}$ is also a feasible solution to the optimization problem (5) associated with the threat point

$$
\left(\frac{1}{2}\left(a_{L B}+\sum_{l \in E} f_{l}\left(x^{*}\right)\right), \frac{1}{2}\left(a_{E E}+\sum_{l \in E} g_{l}\left(x^{*}\right)\right)\right) .
$$

On the other hand, the objective of (5) is equivalent to maximize

$$
\log \left(a_{L B}-\sum_{l \in E} f_{l}\left(x_{l}\right)\right)+\log \left(a_{E E}-\sum_{l \in E} g_{l}\left(x_{l}\right)\right)
$$

Since $x^{*}$ is the optimal solution of (5) associated with the threat point $\left(a_{L B}, a_{E E}\right)$, so we have

$$
\frac{\sum_{l \in E} \nabla f_{l}\left(x^{*}\right)}{a_{L B}-\sum_{l \in E} f_{l}\left(x^{*}\right)}+\frac{\sum_{l \in E} \nabla g_{l}\left(x^{*}\right)}{a_{E E}-\sum_{l \in E} g_{l}\left(x^{*}\right)}=0
$$

When the threat point is

$$
\left(\frac{1}{2}\left(a_{L B}+\sum_{l \in E} f_{l}\left(x^{*}\right)\right), \frac{1}{2}\left(a_{E E}+\sum_{l \in E} g_{l}\left(x^{*}\right)\right)\right),
$$

we should maximize

$$
\begin{aligned}
& F(x)=\log \left(\frac{1}{2}\left(a_{L B}+\sum_{l \in E} f_{l}\left(x^{*}\right)\right)-\sum_{l \in E} f_{l}(x)\right) \\
& +\log \left(\frac{1}{2}\left(a_{E E}+\sum_{l \in E} g_{l}\left(x^{*}\right)-\sum_{l \in E} g_{l}(x)\right)\right.
\end{aligned}
$$

The gradient of $F(x)$ is

$$
\begin{aligned}
& \nabla F(x)=\frac{\sum_{l \in E} \nabla f_{l}(x)}{\frac{1}{2}\left(a_{L B}+\sum_{l \in E} f_{l}\left(x^{*}\right)\right)-\sum_{l \in E} f_{l}(x)} \\
& +\frac{\sum_{l \in E} \nabla g_{l}(x)}{\frac{1}{2}\left(a_{E E}+\sum_{l \in E} g_{l}\left(x^{*}\right)-\sum_{l \in E} g_{l}(x)\right.}
\end{aligned}
$$

We should note that $x=x^{*}$ will yield $\nabla F(x)=0$. Due to the fact that there is only one optimal solution for each Nash Bargaining problem, accordingly, $x^{*}$ is also the Nash Bargaining solution associated with the threat point

$$
\left(\frac{1}{2}\left(a_{L B}+\sum_{l \in E} f_{l}\left(x^{*}\right)\right), \frac{1}{2}\left(a_{E E}+\sum_{l \in E} g_{l}\left(x^{*}\right)\right)\right) .
$$

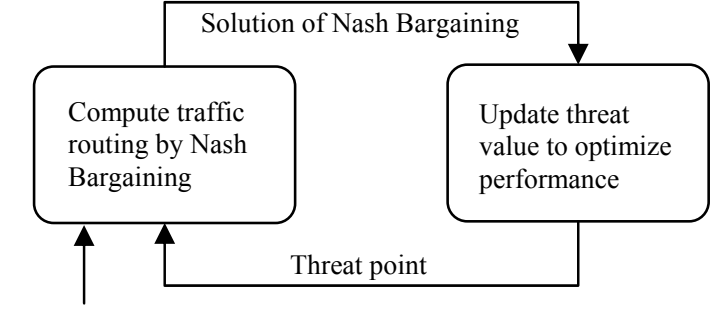

Initial threat point

Fig.1 Procedure of repeated Nash Bargaining

Theorem 3: If each player optimizes its performance selfishly by changing its threat value under Mechanism 1, traffic routing will be constant as if the threat point was never changed.

Proof:

During $k^{\text {th }}$-iteration, each player can at most reduce its threat value to be

$$
\frac{1}{2}\left(a_{L B}^{(k)}+\sum_{l \in E} f_{l}\left(x^{(k)}\right)\right) \text { and } \frac{1}{2}\left(a_{E E}^{(k)}+\sum_{l \in E} g_{l}\left(x^{(k)}\right)\right)
$$

which are larger than

$$
\sum_{l \in E} f_{l}\left(x^{(k)}\right) \text { and } \sum_{l \in E} g_{l}\left(x^{(k)}\right)
$$

respectively. So the reduction of threat value will not lead to negotiation broken and an infinite cost to the players.

According to lemma 1, player $L B$ will reduce its threat value to be $\frac{1}{2}\left(a_{L B}^{(k)}+\sum_{l \in E} f_{l}\left(x^{(k)}\right)\right)$ and player $E E$ will reduce its threat value to be $\frac{1}{2}\left(a_{E E}^{(k)}+\sum_{l \in E} g_{l}\left(x^{(k)}\right)\right)$ during $k^{\text {th }}$-iteration.

Also, from Lemma 2, we know that the optimal solution of Nash Bargaining will be the same as if the threat point was never changed.

Corollary 1: If we set the threat point at any points on the line connecting threat point $\left(a_{L B}, a_{E E}\right)$ and optimization solution of (5) corresponding to this threat point, it will derive the same

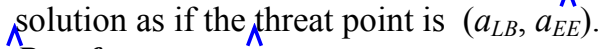

\section{Proof:}

This corollary can be proven in the same methed as temma 2. Due to space limitation, we omit the detail of proof here.

Corollary 1 tells us that all the points on the line eennecting ( $L P \equiv E E_{\text {worst }}$ ) will lead to a fair solution by setting it as the 祀至point of (5).

\section{Conversion to Convex Optimization Form}

The optimization problem (5) is not in a convex optimization form. To solve it more efficiently, we should convert it into the form of standard convex optimization form without changing its solution.

Theorem 4: If the optimization problem in (5) is feasible, its solution will be the same as problem (14):

maximize $\log \left(a_{L B}-t_{L B}\right)+\log \left(a_{E E}-t_{E E}\right)$

subject to: The constraints in (5)

$$
\begin{aligned}
& t_{L B} \geq \sum_{l \in E} f_{l}\left(x_{l}\right) \\
& t_{E E} \geq \sum_{l \in E} g_{l}\left(x_{l}\right)
\end{aligned}
$$

Proof: 
To maximize objective of (14), the variable $t_{L B}$ and $t_{E E}$ should be as little as possible. Therefore, $t_{L B}=\sum_{l \in E} f_{l}\left(x_{l}\right)$ and $t_{E E}=\sum_{l \in E} g_{l}\left(x_{l}\right)$ must be satis $\overline{\overline{\bar{F}}}$ in the optimal solution. So that problem (14) is equivalent to maximize

$$
\log \left(a_{L B}-\sum_{l \in E} f_{l}\left(x_{l}\right)\right)+\log \left(a_{E E}-\sum_{l \in E} g_{l}\left(x_{l}\right)\right)
$$

When the problem in (5) is feasible, we have

$$
\sum_{l \in E} f_{l}\left(x_{l}\right) \leq a_{L B} \text { and } \sum_{l \in E} g_{l}\left(x_{l}\right) \leq a_{E E} .
$$

In this case, the variable maximizing the objective of (5) also maximize (15), because $\log (\cdot)$ is an increasing function of its argument on $\boldsymbol{R}^{+}$.

Theorem 5: If $f_{l}(\cdot)$ and $g_{l}(\cdot)$ are both convex functions, problem (14) is a convex optimization problem.

Proof:

Theorem 5 can be verified easily by checking that it does satisfy the definition of convex optimization.

Theorem 4 and theorem 5 guarantee the Nash Bargaining solution can be solved efficiently.

\section{CASE StUdies}

In this section, we will present two case studies of our methods. We will first apply our method in a simple parallel links network to realize a fair tradeoff between load balance and energy efficiency. It not only presents how our method works but also verifies its correctness. We will then apply our method in a realistic network NSFNET to show its practicability. All the computations are carried out on a computer with Duo-Core 2.20 $\mathrm{GHz}$ intel CPU using CVX1.22 [18].

\section{A. Case Study in a Simple Network}

In this subsection, we apply our model in a simple parallel link network as shown in Fig. 2 to illustrate how our method works. In this network, there are 3 parallel links from node $s$ to node $d$ with capacity of 1000,2000 and 1500 (units) respectively. We also assume that there is a demand from $s$ to $d$ for 900 units of capacity in the network.

As discussed in Sections III and IV, we set the cost of load balance and energy efficiency to be $f_{l}(x)=\frac{x}{c_{l}-x}$ and $g_{l}(x)=\left(\frac{x}{1000}\right)^{1.5}$ for all $l \in E$. From the optimization problem (1), we can find that the best case cost for load balance is 0.6783 and this solution corresponds to the worst case cost for energy efficiency of 0.6223 . Similarly, by solving problem (2) we can also determine the best case cost for energy efficiency and worst case cost for load balance to be 0.4930 and 0.8555 respectively. Fig. 3 shows the Pareto frontier and such two limitation scenarios. Obviously, such two cases are the bounds of Pareto frontier. Note that we choose to use this simple example where the costs for load balance and energy efficiency have comparable values in order to show (below) that even in such a case, our method is more useful than the conventional method that uses an AOF.

To get a fair solution, we set the threat point to be $(0.8555$,

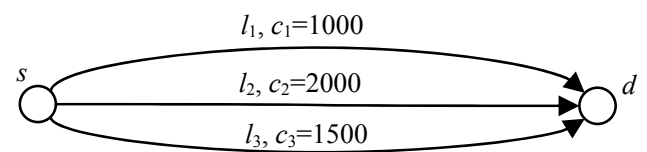

Fig. 2 Parallel link network we consider in case study

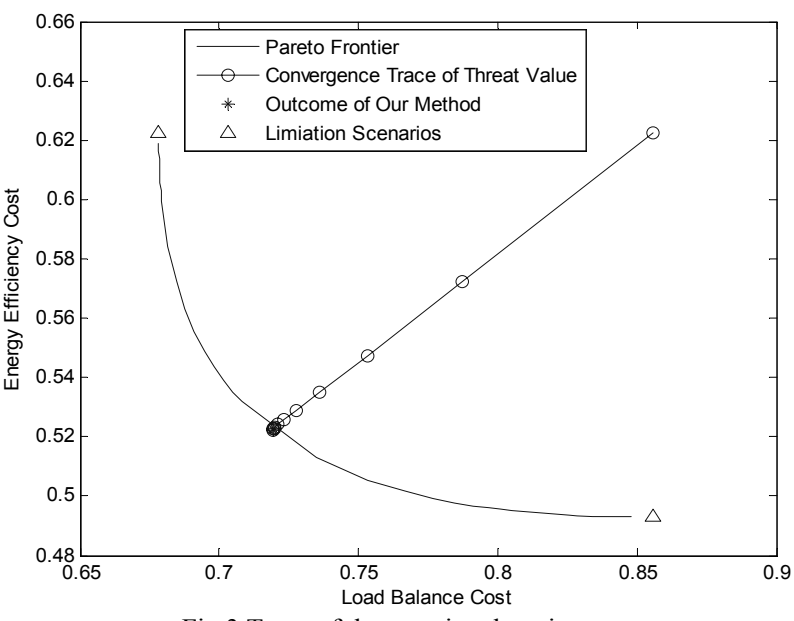

Fig.3 Trace of threat point changing

0.6223) as shown in Fig.3 and use the optimization problem (15) to solve the Nash Bargaining problem. In the solution, the traffic carried by each link is $\left(x_{1}, x_{2}, x_{3}\right)=(151.6013,442.1033$, 306.2954) and the cost for load balance and energy efficiency are 0.7191 and 0.5227 respectively. We should note the following relationship:

$$
\frac{0.8555-0.7191}{0.8555-0.6783}=\frac{0.6223-0.5227}{0.6223-0.4930}=77 \%
$$

It means that each player improve its performance with the same percentage of its optimization space, which shows that the solution derived by our method is fair to both objectives.

To verify the correctness of our mechanism design for the repeated threat value game, we also apply it in the simple network shown in Fig. 2 with the initialized threat point $\left(L B_{\text {worst }}\right.$, $\left.E E_{\text {worst }}\right)$. Fig. 3 shows how the threat point moves hen the iteration executed and the performance of each player in each iteration. We find that if the initial threat point is fixed, the moving of threat point will not impact the solution of the Nash Bargaining and the threat point will convergent to the pair solution point on Pareto frontier through a linear trace. The previous phenomenon verifies the correctness of Lemma 2 and the later one suggests that the corollary is right.

If one uses the classic AOF method to get a tradeoff for this problem, she may set the same weight to both objectives because they have the same order of magnitude. In this case, the resulting cost for load balance is 0.7081 and the cost for energy efficiency is 0.5318. Though this solution is Pareto efficient, energy efficiency obtains less performance improvement $(70 \%)$ than load balance (83\%).

In our method, we set the performance threshold of each objective to be its worst case performance. If one uses the conventional approach whereby one objective is treated as a constraint, she obviously cannot use the worse-case performance as the threshold value for any of the objective, but what makes such an approach difficult is that she also won't know which other threshold value is the most appropriate. She 


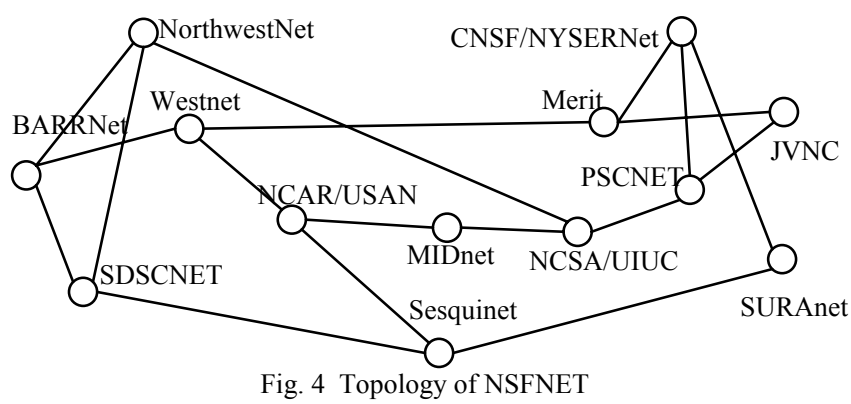

may choose a medium value between the worst and best cases for example, but such a choice is ad hoc by the best and cannot be considered fair.

\section{B. Case Study in a NSFNET}

In this section, we will use our proposed method to realize a fair tradeoff between load balance and energy efficiency in the NSFNET backbone network (shown in Fig. 4). In this network, each link is bidirectional and with loss of generality, we assume that the capacity of each link is $45 \mathrm{Mbps}$ (such capacity is offered by NSFNET during 1992 and 1995 [19] and was chosen to simplify our computation only). To route the demand in the network, we find two link disjointed routes between each node pairs and we add a 10Mpbs demand between every pair of the six supercomputer sites (SDSCNET, NCSA, CNSF, PSCNET, JVNC and NCAR). In order to show that our method can derive a fair solution even each objective has different order of magnitude, we set the energy curve of link $l$ to be $g_{l}(x)=\left(\frac{x}{45}\right)^{1.5}$.

In this case study scenario, the best case cost for load balance is 18.3378 while the worst case cost is $2.9597 \times 10^{7}$, which is much worse than the best case. For energy efficiency, the best and worst case costs are 5. 42 and 5.8313 respectively. This means that the optimization space of load balance is much larger than that of energy efficiency.

Note that if the conventional approach based AOF were to be used in this case, one would not know how to set an appropriate weight for each objective. More specifically, if she uses more or less the same weights for the two objectives, the load balance will get much more performance improvement than energy efficiency, because it has a larger optimization space than energy efficiency, which would be unfair to energy efficiency. In short, a fair solution is difficult to obtain by using the AOF method.

In our method, we set the threat point to be $\left(2.9597 \times 10^{7}\right.$, 5.8313 ) to derive a fair solution. In the solution, the cost of load balance is 261.0334 while the cost of energy efficiency is very close to 5.4200 (the accuracy is to $10^{-4}$ ). This represents $99.999 \%$ optimization space.

\section{CONCLUSION}

In this paper, we have studied how to achieve a fair tradeoff between load balance and energy efficiency in traffic engineering. Different from the traditional multi-objective optimization methods which either construct an aggregate objective function (AOF) or treat one of the objectives as a constraint of the problem, we have analyzed such a problem from a game theoretic perspective. More specifically, we have treated the two objectives as two virtual players in a so-called threat value game, who negotiate with each other in order to achieve an agreement under the Nash Bargaining framework. In such a game, each player can announce its threat value to optimize its performance and our analysis have shown that the number of Nash equilibriums can be infinite and each player determines its threat value will prevent an agreement, so as to induce an infinite cost to both of them. To avoid such an undesirable outcome, we have designed a mechanism that can not only reach an agreement but also lead to a fair tradeoff between load balance and energy efficiency. In addition, find out all the threat points which can be used to get the fair tradeoff solution.

Although this work focuses on achieving a fair tradeoff between load balance and energy efficiency in traffic engineering, it also provides some useful insights into the other multi-objective optimization problems.

\section{REFERENCES}

[1] D. Awduche, "MPLS and Traffic Engineering in IP Networks," IEEE Communications Magazine, vol. 37, no. 12, pp. 42-47, Dec. 1999.

[2] B. Fortz and M. Thorup, "Internet Traffic Engineering by Optimizing OSPF Weights", in Proc. $19^{\text {th }}$ IEEE Conf. on Computer Communications (INFOCOM), 2000, pp. 519-528

[3] S. Secci, K. Liu, K. Rao, and B. Jabbari, "Resilient Traffic Engineering in a Transit-Edge Separated Internet Routing," in Proc. IEEE ICC, 2011.

[4] G. Shrimali, A. Akella and A. Mutapcic, "Cooperative Interdomain Traffic Engineering Using Nash Bargaining and Decomposition", IEEE Trans. on Netw. vol. 18, no. 2, pp. 341-352, April 2010

[5] M. Andrews, A.F. Anta, L. Zhang and W. Zhao, "Routing for Energy Minimization the Speed Scaling Model", in Proc, $29^{\text {th }}$ IEEE Conf. on Computer Communication (INFOCOM), 2010, pp. 1-9

[6] E. Yetginer and G.N. Rouskas, "Power Efficient Traffic Grooming in Optical WDM Networks", in Proc. $52^{\text {th }}$ IEEE Global Telecommunication Conf. (GLOBECOM), 2009, pp. 1-6

[7] A. Wierman, Lachlan L. H. Andrew, and Ao Tang. "Power-aware speed scaling in processor sharing systems", in Proc. $28^{\text {th }}$ IEEE Conf. on Computer Communication (INFOCOM), 2009, pp. 1-9

[8] Enhanced Intel Speed Step Technology for the Intel Pentium M processor. Intel White Paper 201170-001, 2004

[9] R. E. Steuer. Multiple Criteria Optimization: Theory, Computation, and Application. New York: John Wiley \& Sons, Inc. ISBN 0-471-8846-X, 1986.

[10] D. Awduche, J. Malcolm, J. Agogbua, M. O’Dell, and J.McManus, "RFC 2702: Requirements for Traffic Engineering Over MPLS," September 1999

[11] M. Xia, M. Tornatore, Y. Zhang, P. Chowdhury, C.U. Martel and B. Mukherjee. "Green Provision for Optical WDM Networks", IEEE Jour. of Sele. Top. in Quan. Elec. vol. 17, no. 2, pp. 437-445, Match 2011

[12] S. Seetharaman, V. Hilt, M. Hofmann, and M. Ammar, "Resolving Cross-Layer Conflict between Overlay Routing and Traffic Engineering", IEEE/ACM Tran. Netw. vol.17, pp 1964 - 1977, 2009

[13] L. Qiu, R. Y. Yang, Y. Zhang, and S. Shenker, "On selfish routing in internet-like environments," in Proc. ACM SIGCOMM, 2003, pp. 151-162.

[14] Y. Liu, H. Zhang, W. Gong, and D. Towsley, "On the interaction between overlay routing and traffic engineering," in Proc. IEEE INFOCOM, 2005, pp. 2543-2553.

[15] W. Jiang, Z.S. Rui, J.Rexford, M. Chiang, "Cooperative Content Distributed and Traffic Engineering in an ISP Network", in Proc. SIGMETRICS/Performance, 2009, pp. 1-12, June 2009.

[16] M.D. Davis. Game Theory, A Nontechnical Introduction. New York: Dover Publications. 1983.

[17] J.F. Nash, "The bargaining problem," Econometrica, vol. 28, pp. 155-162, 1950.

[18] M. Grant and S. Boyd, "cvx Users' Guide for cvx version 1.22", http://cvxr.com/cvx/cvx_usrguide.pdf

[19] L.M. David and H. Braun, "The NSFNET Backbone Network", http://www.eecis.udel.edu/ mills/database/papers/bone/bone.pdf 